\title{
IMPACTO DO DESFECHO NEONATAL EM PUÉRPERAS DE RECÉM-NASCIDOS PORTADORES DE ANOMALIA CONGÊNITA*
}

Julyane Vasconcelos Oliveira', Flavia Westphal², Anelise Riedel Abrahäo ${ }^{3}$

${ }^{1}$ Acadêmica de Enfermagem. Universidade Federal de São Paulo. São Paulo-SP-Brasil.

${ }^{2}$ Enfermeira. Mestranda em Enfermagem. Universidade Federal de São Paulo. São Paulo-SP-Brasil.

${ }^{3}$ Enfermeira. Doutora em Enfermagem. Professora da Universidade Federal de São Paulo. São Paulo-SP-Brasil.

RESUMO: Este estudo teve por finalidade identificar em puérperas, sinais de estresse decorrentes do processo de enfrentamento da gestação de um feto portador de anomalia congênita. Trata-se de um estudo transversal, realizado no ambulatório de medicina fetal da Universidade Federal de São Paulo, entre janeiro e abril de 2014. Para este estudo foram utilizados dois instrumentos de coleta de dados: um formulário de identificação sociodemográfica e de antecedentes reprodutivos e a Escala do Impacto do Evento- Revisada, aplicados a 24 puérperas. Foram identificados sinais de estresse em $62,5 \%$ das puérperas estudadas, sendo que destas, $41,7 \%$ gestaram fetos inviáveis. Quanto ao período gestacional do diagnóstico fetal, verificou-se que $75 \%$ das mulheres o receberam no $2^{\circ}$ trimestre de gestação e do total, 37,5\% apresentaram alta probabilidade de ter transtorno de estresse pós-traumático. Assim, podemos supor que o diagnóstico precoce permite assistência de maior qualidade a gestante/casal/família, favorecendo evolução pós-parto com menor estresse.

DESCRITORES: Anormalidades congênitas; Período pós-parto; Transtornos de estresse traumático; Enfermagem materno-infantil.

\section{NEONATAL OUTCOME IMPACT IN PUERPERAL MOTHERS OF NEWBORNS WITH CONGENITAL ANOMALY}

ABSTRACT: The aim of this study was to identify signs of stress in puerperal women arising from the coping process of gestation of a fetus with a congenital anomaly. This was a cross-sectional study developed at the fetal medicine outpatient clinic of the Federal University of São Paulo between January and April 2014. Two data collection instruments were used for this study: a questionnaire on sociodemographic identification and reproductive history, and the Revised Impact of Event Scale, which were applied to 24 puerperal women. Signs of stress were identified in $62.5 \%$ of the study subjects; of these, $41.7 \%$ gestated a non-viable fetus. In regard to the gestational period of diagnosis of the fetus, $75 \%$ of the women received diagnosis in the second trimester of pregnancy, and of the total number of subjects, $37.5 \%$ presented high likelihood of having post-traumatic stress disorder. Thus, early diagnosis enables higher quality care to pregnant women/couples/families, thereby encouraging postpartum development with less stress.

DESCRIPTORS: Congenital abnormalities; Postpartum period; Traumatic stress disorders; Maternal-child nursing.

\section{IMPACTO DEL DESHACER NEONATAL EN PUÉRPERAS DE RECIÉN NACIDOS PORTADORES DE ANOMALÍA CONGÉNITA}

RESUMEN: Este estudo tuvo por finalidad identificar en puérperas señales de estrés decurrientes del proceso de afrontamiento de la gestación de un feto portador de anomalía congénita. Es un estudio transversal, realizado en el ambulatorio de medicina fetal de Universidad Federal de São Paulo, entre enero y abril de 2014. Para el estudio fueron utilizados dos instrumentos para recoger datos: un formulario de identificación sociodemográfica y de antecedentes reproductivos y la Escala del Impacto de Evento- Revisada, aplicados a 24 puérperas. Fueron identificados señales de estrés en $62,5 \%$ de las puérperas estudiadas, siendo que de estas, $41,7 \%$ gestaron fetos inviables. Acerca del periodo gestacional del diagnóstico fetal, se verificó que $75 \%$ de las mujeres lo tuvieron en el segundo trimestre de gestación y del total, $37,5 \%$ presentaron alta probabilidad de tener trastorno de estrés postraumático. Así, es posible suponer que el diagnóstico precoz permite asistencia de mayor cualidad a gestante/pareja/ familia, favorecendo evolución posparto con menos estrés. DESCRIPTORES: Anormalidades congénitas; Periodo posparto; Trastornos de estrés traumático; Enfermería materno infantil.

*Artigo extraído da monografia de conclusão de curso da Graduação em Enfermagem intitulado: "O impacto do desfecho neonatal em puérperas de recém-nascidos portadores de anomalia congênita". Universidade Federal de São Paulo, 2014. 


\section{INTRODUÇÃO}

Na literatura há diversas definições para anomalia congênita, malformação congênita ou defeito congênito. Considerando a origem do termo congênito (nascido com), uma possível definição seria a de qualquer anormalidade estrutural ou funcional presente ao nascimento devido a causa genética, ambiental ou multifatorial ${ }^{(1)}$.

Dentre as malformações congênitas temos as classificadas como viáveis, por exemplo, gastrosquise, espinha bífida, hidrocefalia, e aquelas que são consideradas incompatíveis com a vida extrauterina, sendo as mais comuns a anencefalia, a agenesia renal bilateral e o body stalk ${ }^{(2)}$.

A Organização Mundial de Saúde (OMS) traz que as anomalias congênitas afetam aproximadamente uma a cada 33 crianças e resultam em aproximadamente 3,2 milhões de deficiências todos os anos. Em 2010, a anomalia congênita foi considerada causadora de 270 mil mortes neonatais em 193 países, o que equivale a $8,7 \%$ das causas de mortes neonatais no mundo ${ }^{(3)}$. Já no Brasil, é a segunda maior responsável pelo óbito infantil, $18 \%$ do número total, atrás apenas das afecções no período perinatal( ${ }^{(4)}$. Esses dados mostram a relevância do tema e a importância de sua abordagem para assistência em saúde.

Historicamente, passamos de uma época em que a descoberta de anomalias fetais só seria possível ao nascimento, para outra na qual o avanço da ciência e tecnologia permitiu a detecção intrauterina de diversos defeitos congênitos, possibilitando, dessa forma, a tomada de condutas em busca do bem estar materno e fetal.

Assim, a medicina fetal iniciou seu desenvolvimento revolucionando e remodelando a obstetrícia. No início da década de 70, o encontro casual de importantes inovações como o aperfeiçoamento de técnicas, ensaios enzimáticos, cariotipagem de células fetais, determinação de metabólitos no líquido amniótico e o desenvolvimento dos aparelhos de ultrassonografia, acabou por levar a remodelação do pré-natal aproximando-se de como o conhecemos atualmente ${ }^{(5)}$.

O desenvolvimento tecnológico na avaliação pré-natal levou a uma significativa melhora na capacidade de diagnóstico de um grande número de patologias congênitas e anomalias do desenvolvimento. A ultrassonografia e a genética trabalhando em conjunto foram os pilares dessa evolução na capacidade terapêutica e, por conseguinte, do avanço no manejo obstétrico.

A medicina fetal se desenvolveu de fato no Brasil entre a década de 80 e 90 com a utilização de técnicas de avaliação do bem estar fetal e aperfeiçoamento das sondas transvaginais de ultrassonografia, ecocardiografia fetal, ultrassonografia tridimensional, os quais permitiram diagnóstico precoce de anomalias ${ }^{(6)}$.

Assim, com a evolução do diagnóstico pré-natal, a detecção das anomalias se tornou uma realidade que muitas vezes favorece o desenvolvimento fetal por meio da implementação de recursos terapêuticos. Com este intuito, várias técnicas de abordagem fetal ainda vêm sendo desenvolvidas e introduzidas no cuidado, como a intervenção intrauterina clássica, a paliativa e a terapêutica definitiva, sendo a última indicada para situações específicas, em que se deve avaliar o risco-benefício da correção ${ }^{(7)}$.

Entretanto, estudos mostram a existência de malformações que não se beneficiam do tratamento durante o período pré-natal, sendo de suma importância o conhecimento do diagnóstico antes do nascimento para o preparo de uma abordagem neonatal imediata, a qual muitas vezes definirá a possibilidade de sobrevida do feto e viabilizará um prognóstico à criança.

Outro enfoque deve ser dado aos casos em que se verificam diagnósticos complexos, como o de inviabilidade fetal. Nesta situação, o diagnóstico pré-natal permite ao casal optar pela solicitação de interrupção voluntária da gestação.

Contudo, em todas as situações abordadas devem ser valorizadas as informações prestadas ao casal e a família, bem como as repercussões emocionais desses diagnósticos, que podem levar ao desenvolvimento do Transtorno do Estresse Pós-Traumático (TEPT). De acordo com a American Psychiatric Association, o transtorno do estresse pós-traumático pode ser definido como um conjunto de sintomas associados a um evento traumatizante ${ }^{(8)}$. Estudos mostram que sintomas de TEPT são observados com frequência após a interrupção da gestação de fetos inviáveis, principalmente se for realizada em idade gestacional avançada ${ }^{(9-10)}$.

O fato de o filho esperado ser substituído por um com diagnóstico de malformação provoca, por si só, uma reação de sofrimento e angústia, 
podendo esta ser bastante intensificada quando a malformação é letal. Conseguir lidar com o prognóstico de malformação depende de diversos fatores como a estrutura emocional do casal e da própria família, bem como dos cuidados de saúde especializados e da assistência multiprofissional disponibilizada.

Com toda a evolução da medicina e da tecnologia para auxiliar a equipe de saúde, o diagnóstico está inestimavelmente mais confiável quanto às anomalias que o feto possui e/ou possa desenvolver futuramente. Isso torna fundamental o papel dos profissionais de saúde, em especial do enfermeiro, no acompanhamento de todo esse processo com a gestante, buscando sempre orientar, acolher e amparar.

Dessa forma, para que a gestante percorra o processo, é fundamental o acompanhamento multiprofissional, com ênfase no suporte psicoeducativo para que ela entenda o diagnóstico e supere cada uma das fases do "luto" pelo filho idealizado, aprendendo a lidar com a situação sem "adoecer"(11).

É de grande importância compreender a relação entre a presença de malformações fetais e o impacto emocional desta condição nas puérperas. Portanto, visando conhecer melhor esta problemática, este estudo teve por objetivo verificar os sinais de estresse em puérperas de fetos portadores de anomalias congênitas.

\section{MÉTODO}

Foi realizado um estudo prospectivo transversal, com amostragem por conveniência consecutiva, de abordagem quantitativa sobre os aspectos emocionais, por meio da identificação dos sinais de estresse, em puérperas que geraram fetos portadores de anomalia congênita. O estudo foi realizado no ambulatório de medicina fetal da Universidade Federal de São Paulo (UNIFESP), um centro de atendimento a pré-natal de alto risco, que conta com uma equipe multiprofissional.

A coleta de dados foi realizada no período de janeiro a abril de 2014. Foram incluídas na amostra mulheres que realizaram o pré-natal no ambulatório de medicina fetal da UNIFESP, que gestaram fetos portadores de anomalias e que estavam, no mínimo, no sétimo dia de pós-parto ou pós-interrupção - nos casos de fetos inviáveis.
Foram excluídas do estudo, mulheres analfabetas que não poderiam responder ao questionário.

A amostra foi composta por 24 participantes, sendo 12 puérperas de fetos viáveis e 12 de fetos inviáveis. Foram utilizados dois instrumentos, o primeiro, um formulário elaborado especificamente para este estudo, que continha perguntas fechadas que contemplava a identificação da mulher, dados demográficos, socioeconômico e histórico gineco-obstétrico (número de gestações, de partos e de abortos, via de parto, presença de comorbidades, entre outros). As informações foram obtidas através do prontuário das pacientes e completadas na entrevista.

O segundo instrumento foi a Escala do Impacto do Evento de Horowitz - Revisada (IES-R) por Weiss e traduzida para o português por Caiuby ${ }^{(12)}$. Trata-se de uma escala utilizada no rastreio de sintomatologia do transtorno de estresse pós-traumático e, neste estudo, foi utilizada para avaliar a presença de sintomatologia nas puérperas que receberem diagnóstico de anomalia fetal na gestação. Essa escala é do tipo likert na qual o indivíduo responde os itens reportando-se aos seus sentimentos nos sete dias anteriores à aplicação do instrumento e possui 22 itens divididos em três subescalas: evitação, intrusão e hiperestimulação, contemplando os critérios do Manual Diagnóstico e Estatístico de Transtornos Mentais $4^{\circ}$ Ed (DSM-IV) quanto aos sintomas do transtorno de estresses póstraumático ${ }^{(8)}$.

A análise das respostas é obtida através do cálculo do escore de cada subescala e o escore total é soma desses escores. Para cada questão a pontuação varia de 0 a 4 pontos e o cálculo do escore é obtido por meio da média dos itens que compõem as subescalas, desconsiderando-se as questões não respondidas.

Neste instrumento os resultados que excedam 24 pontos podem ser bastante significativos, pois em pacientes que apresentem pelo menos alguma sintomatologia, o TEPT passa a ser uma preocupação clínica. Resultados acima de 33 pontos representam o melhor ponto de corte para o rastreio e provável diagnóstico de TEPT, devidamente complementado pela avaliação clínica. Já um resultado superior a 37 pontos é considerado alto o suficiente para o comprometimento do sistema imunológico ${ }^{(13)}$. 
A Escala do Impacto do Evento - revisada foi aplicada em um local calmo e o aplicador era responsável pela leitura das questões. Os resultados obtidos, juntamente com os do formulário, foram armazenados no programa Excel. As variáveis estão apresentadas com valores de média, desvio padrão, medianas e variação com valores mínimos e máximos. Os resultados são apresentados em números absolutos e em percentual.

Este estudo seguiu as normas para pesquisa envolvendo seres humanos definidas na Resolução n. 466 do Conselho Nacional de Saúde, tendo sido aprovado pelo Comitê de Ética e Pesquisa da Universidade Federal de São Paulo - UNIFESP, sob o n ${ }^{\circ}$ 25771913.4.0000.5505 (14).

\section{RESULTADOS}

A idade das mulheres que participaram desse estudo variou entre 20 e 42 anos e os resultados permitiram identificar os seguintes dados epidemiológicos e obstétricos apresentados nas Tabelas 1 e 2.

Foram analisados ainda alguns hábitos de vida, obtendo-se que $41,1 \%$ das mulheres consideramse católicas, 33,3\% evangélicas e o restante entre não praticantes e outras religiões. Quanto ao hábito de fumar e ingerir bebidas alcoólicas, $95,8 \%$ afirmaram não fumar, $25 \%$ faziam uso de bebidas alcoólicas socialmente e os $75 \%$ restantes não consumiam álcool. Por fim, quanto ao uso de drogas ilícitas, 8,3\% disse já ter feito uso e 91,7\% não se declararam usuárias.

Considerando os resultados sobre antecedentes de malformação observaram-se os seguintes dados visualizados na Figura 1.

Em relação à gestação analisada, não houve relatos de gravidez não desejada, sendo relativamente semelhante o número de planejadas e não planejadas (41,7 e 58,3\%, respectivamente). Quanto ao início do pré-natal, verificou-se que $87,5 \%$ das mulheres iniciaram no primeiro trimestre da gestação, $12,5 \%$ no segundo trimestre, não havendo casos de início no terceiro trimestre. Identificou-se que $75 \%$ das mulheres tiveram o diagnóstico de malformação no segundo trimestre da gestação e apenas $25 \%$ no primeiro. Já em relação à idade gestacional de admissão no ambulatório de medicina fetal da
Tabela 1 - Perfil epidemiológicodas mulheresquegestaram filhos com anomalia congênita. São Paulo-SP-Brasil, 2014

\begin{tabular}{|c|c|c|}
\hline Variáveis & $\mathbf{n}$ & $\%$ \\
\hline \multicolumn{3}{|l|}{ Estado Civil } \\
\hline Casada/União estável & 20 & 83,3 \\
\hline Solteira & 4 & 16,7 \\
\hline \multicolumn{3}{|l|}{ Etnia } \\
\hline Branca & 13 & 54,1 \\
\hline Não Branca & 11 & 45,9 \\
\hline \multicolumn{3}{|l|}{ Situação de Moradia } \\
\hline Própria & 9 & 37,5 \\
\hline Alugada & 13 & 54,2 \\
\hline Emprestada & 2 & 8,3 \\
\hline \multicolumn{3}{|l|}{ Naturalidade } \\
\hline São Paulo & 15 & 62,5 \\
\hline Outros & 9 & 37,5 \\
\hline \multicolumn{3}{|l|}{ Escolaridade } \\
\hline Ensino fundamental & 4 & 16,7 \\
\hline Ensino médio & 18 & 75 \\
\hline Ensino superior & 2 & 8,3 \\
\hline \multicolumn{3}{|l|}{ Ocupação } \\
\hline Dona de casa/desempregada & 9 & 37,5 \\
\hline Estudante & 1 & 4,2 \\
\hline Trabalhador formal & 2 & 8,3 \\
\hline Trabalhador informal & 12 & 50 \\
\hline \multicolumn{3}{|l|}{ Provedor da Família } \\
\hline Entrevistado & 2 & 8,3 \\
\hline Companheiro & 7 & 29,2 \\
\hline Ambos & 13 & 54,2 \\
\hline Outros & 2 & 8,3 \\
\hline \multicolumn{3}{|l|}{ Renda familiar } \\
\hline < 1 salário mínimo & 4 & 16,7 \\
\hline$\geq 1$ até 3 salários mínimos & 16 & 66,7 \\
\hline$\geq 3$ até 5 salários mínimos & 3 & 12,5 \\
\hline$\geq 5$ salários mínimos & 1 & 4,1 \\
\hline \multicolumn{3}{|l|}{ Idade } \\
\hline 19 a 34 anos & 19 & 79,1 \\
\hline$\geq 35$ anos & 5 & 20,9 \\
\hline
\end{tabular}

UNIFESP, constatou-se que as mulheres chegaram a sua totalidade no segundo (83,3\%) e no terceiro trimestre $(16,7 \%)$.

Dentre as gestações analisadas observou-se número igual de fetos viáveis e inviáveis durante o período, sendo que apenas $16 \%$ das gestações de inviáveis puderam optar e efetivar a interrupção da gestação, conforme demonstra a Figura 2.

Avaliando a presença de sinais estresse 
através da pontuação obtida na IES-R, conforme observamos na Figura 3 e Tabela 3, identificamos que $62,5 \%$ das mulheres do estudo apresentaram alguma sintomatologia característica do TEPT - pontuação maior que 24 no escore. Ainda identificou-se em 37,5\% (n=9) das puérperas participantes uma pontuação superior a 37, alta o suficiente para causar supressão do sistema

Tabela 2 - Perfil obstétrico das mulheres que gestaram filhos com anomalia congênita. São Paulo-SP-Brasil, 2014

\begin{tabular}{lcc}
\hline Variáveis & $\mathbf{n}$ & $\mathbf{\%}$ \\
\hline $\mathrm{N}^{\mathbf{0}}$ de gestações & & \\
\hline Primigesta & 11 & 45,8 \\
\hline Secundigesta & 8 & 33,3 \\
\hline Tercigesta & 3 & 12,5 \\
\hline Quatro ou mais & 2 & 8,4 \\
\hline $\mathrm{N}^{\circ}$ de partos & & \\
\hline Um parto & 11 & 45,8 \\
\hline Dois partos & 8 & 33,4 \\
\hline Três ou mais & 5 & 20,8 \\
\hline Abortos & & \\
\hline Sim & 6 & 25 \\
\hline Não & 18 & 75 \\
\hline $\mathrm{N}^{0}$ de abortos & & \\
\hline Um & 4 & 66,7 \\
\hline Dois & 2 & 33,3 \\
\hline Tipo do aborto & \\
\hline Precoce & 4 & 66,7 \\
\hline Tardio & 2 & 33,3 \\
\hline
\end{tabular}

imunológico e provável TEPT. Dentre estas, 77,7\% $(n=7)$ eram mães de fetos inviáveis, evidenciando que a ocorrência de trauma é maior quando diagnosticada a inviabilidade fetal.

Não houve correlação significativa entre as pontuações na IES-R e variáveis maternas tais como: idade, número de filhos, escolaridade, estado civil, aspectos socioeconômicos e antecedentes obstétricos.

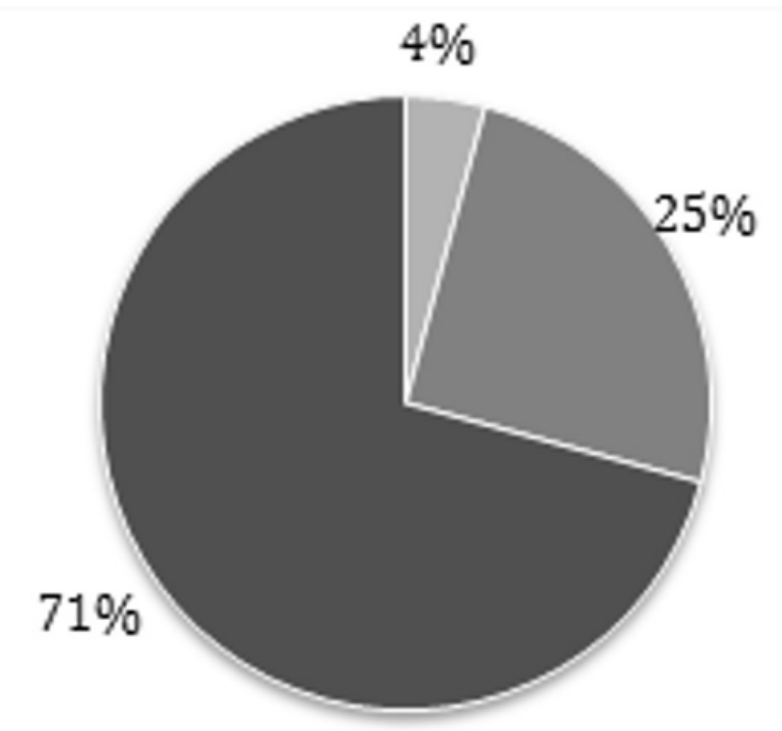

\section{Malformação congênita anterior}

Caso de malformação em familiar

Casos sem antecedentes

Figura 1- Caracterização das mulheres que gestaram filhos com anomalia congênita segundo a presença de antecedentes. São Paulo, SP, Brasil, 2014

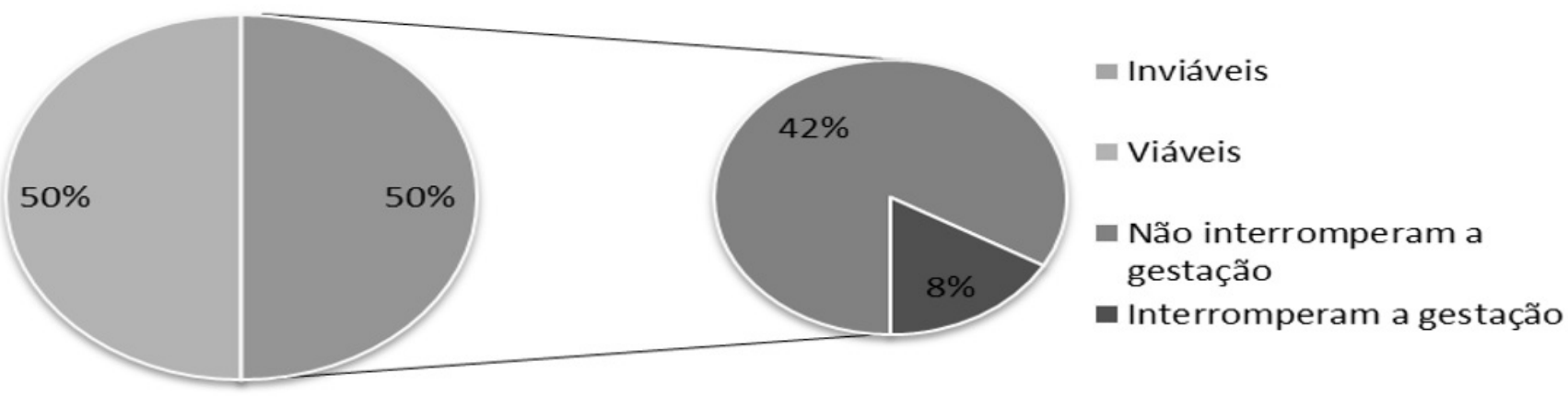

Figura 2- Caracterização das mulheres com fetos portadores de anomalia congênita segundo viabilidade fetal e opção por interrupção voluntária da gestação. São Paulo, SP, Brasil, 2014 


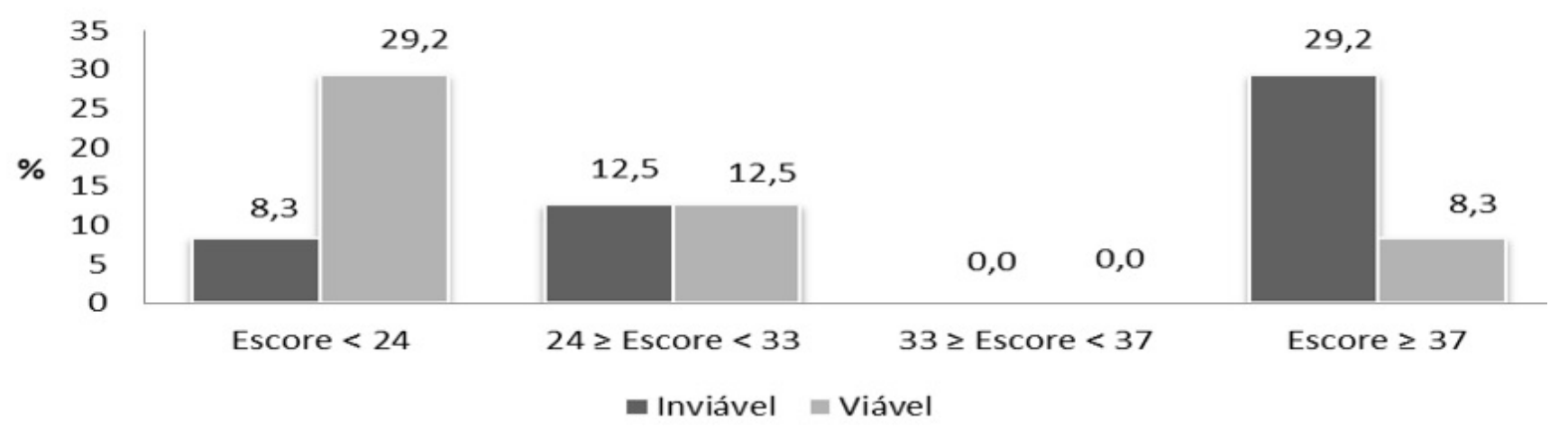

Figura 3- Caracterização das mulheres com fetos portadores de anomalia congênita segundo viabilidade fetal e escore da IES-R. São Paulo, SP, Brasil, 2014

Tabela 2 - Variação do Escore da IES-R entre mulheres que gestaram fetos com malformação viável e malformação inviável. UNIFESP, São Paulo, SP, Brasil, 2014

\begin{tabular}{lccccc}
\hline Viabilidade fetal & $\begin{array}{c}\text { Média do escore } \\
\text { da IES-R }\end{array}$ & Mediana & Mínimo & Máximo & Desvio Padrão \\
\hline Viável & 20,2 & 21 & 2 & 44 & 15,1 \\
\hline Inviável & 37 & 39 & 3 & 62 & 18,6 \\
\hline
\end{tabular}

\section{DISCUSSÃO}

Alguns estudos relatam que a prevalência de depressão pós-parto varia de 3,7\% a 48,6\%, mostrando ser um fator bastante comum entre as puérperas por tratar-se de um período de grandes mudanças fisiológicas e de exigências de um desempenho adequado da função materna ${ }^{(15-16)}$.

Outros estudos relatam que o risco de desenvolver ansiedade e/ou depressão é maior em mulheres que passaram por vivências estressantes nos últimos 12 meses, sendo esse índice ainda maior entre puérperas de recém-nascidos com malformações do que em saudáveis ${ }^{(17-18)}$.

O Manual Diagnóstico e Estatístico de Transtornos Mentais $4^{\circ}$ Ed (DSM-IV) define evento traumático como evento catastrófico que pode envolver morte, lesão real/ameaçada ou ameaça à integridade física de si mesma, sendo caracterizada por medo intenso, impotência ou horror ${ }^{(8)}$. Sendo assim, a vivência de um diagnóstico de malformação congênita pode ser considerada um fator traumático para gestantes e puérperas, pois o momento dessa constatação contradiz o filho idealizado ${ }^{(19-20)}$.

O uso da Escala de Impacto do Evento - Revisada (IES-R) nesta pesquisa, permitiu identificar que a ocorrência de trauma é evidenciada tanto em puérperas de fetos viáveis como inviáveis. Contudo, a associação de transtorno de estresse pós-traumático (TEPT) e pós-parto é bastante recente. Uma revisão da bibliográfica acerca de transtornos psiquiátricos no pós-parto encontrou que a prevalência de TEPT causada por eventos do periparto, como é o caso do diagnóstico de anomalia congênita, varia de $1,5 \%$ a $5,6 \%$.

Outra revisão bibliográfica sobre malformações congênitas mostrou que não somente a mãe, como também o pai precisam obter informações sobre os recursos e auxílios, falar sobre a malformação e principalmente, ter tempo para se ajustar a nova situação ${ }^{(22)}$. Isto é especialmente grave quando se trata de gestação de fetos inviáveis, que ao chegar tardiamente ao serviço fica limitada à opção pelo curso da gestação, prejudicando a possibilidade de um acompanhamento psicológico, social e judicial em um tempo adequado. Essa situação foi observada neste estudo, em que as mulheres foram admitidas no serviço especializado em medicina fetal tardiamente. E para esse grupo, o acompanhamento multiprofissional especializado com condutas e planos de cuidados individualizados é fundamental para o enfrentamento de períodos de dúvidas, em que a aceitação do problema é difícil e muitas vezes traumática ${ }^{(23)}$. 
No presente estudo, verificou-se uma limitação devido ao tamanho amostral. Apesar de ter sido realizada em um ambulatório de referência do estado, que atende uma grande área populacional, houve dificuldade para compor a amostra em virtude do não retorno dessas puérperas para continuidade do acompanhamento. Esse fato permite inferir diversos fatores que podem contribuir para isso, como a distância entre o local de moradia e o ambulatório ou a falta de significado para estas mulheres quanto à importância da continuidade do acompanhamento após o parto ou interrupção.

Seja qual for o motivo, esse dado revela a necessidade de se estabelecer maior vínculo entre a gestante e o serviço de pré-natal a fim de conscientizá-la sobre a continuidade de assistência no período puerperal. O acompanhamento psicoprofilático é fundamental e deverá ser continuado por aproximadamente quatro meses após o parto, garantindo uma evolução satisfatória e um re-encaminhamento (contra referência) seguro para a unidade de menor complexidade da região de moradia desta mulher.

\section{CONSIDERAÇÕES FINAIS}

Este estudo identificou que a gestação de fetos portadores de anomalias congênitas constituiu-se em um importante fator de risco para a criação de um trauma, sendo este mais intensificado frente ao diagnóstico de inviabilidade fetal.

Dada a importância da temática e a fim de obter dados que permitam análises estatísticas mais consistentes, nos próximos estudos sugerese que seja realizada a composição de amostras multicêntricas, como ocorre em outros estudos de síndromes de rara aparição.

\section{REFERÊNCIAS}

1. Moore KL, Persaud TVN, Torchia, MG, Persaud T. The seveloping human: clinically oriented embryology. 9ed .Philadelphia: Saunders/Elsevier; 2009.

2. Consonni EB, Petean EBL. Perda e luto: vivências de mulheres que interromperam a gestação por malformação fetal letal. Cienc. Saude Colet. [Internet] 2013;18(9) [acesso em 15 jan 2014]. Disponível: http:// dx.doi.org/10.1590/S1413-81232013000900021

3. Organización mundial de la salud. Anomalías congénitas: nota descriptiva N³70. [Internet]. [atualizada em jan 2014; acesso em 15 jan 2014]. Disponível: http://www.who.int/mediacentre/ factsheets/fs370/es/

4. Painel de monitoramento da mortalidade Infantil e Fetal [Internet]. Brasília: Sistema de Informações sobre Mortalidade; [acesso em 15 jan 2013]. Disponível: http://svs.aids.gov.br/dashboard/mortalidade/infantil. show.mtw

5. Löwy I. Detectando más-formações, detectando riscos: dilemas do diagnóstico pré-natal. Horiz. antropol.. 2011;17(35):103-28.

6. Gollop TR. Aborto por anomalia fetal. Bioética. [Internet] 2010;2(1) [acesso em 10 jan 2014]. Disponível: http://revistabioetica.cfm.org.br/index. php/revista_bioetica/article/view/94/100

7. Sbragia L. Intrauterine fetal abnormalities therapy. Rev. Bras. Ginecol. Obstet. 2010;32(1):47-7.

8. Diagnostic and statistical manual of mental disorders, text revised, DSM-IV TR. 4 th. Washington; 2000.

9. Brunner MAC. Prevalência da depressão pós-parto entre mulheres assistidas no ambulatório de pós-natal do Instituto Fernandes Figueira-Fiocruz [tese]. Rio de Janeiro (RJ): Instituto Fernandes Figueira; 2011.

10. Pereira PK, Lima LA, Legay LF, Santos JFC, Lovisi GM. Malformação congênita do bebê e risco de transtornos mentais maternos durante o período gravídicopuerperal: uma revisão sistemática. Cad. Saude Colet. 2011;19 (1):2-10.

11. Santos MM, Böing E, de Oliveira ZAC, Crepaldi MA. Diagnóstico pré-natal de malformação incompatível com a vida: implicações psicológicas e possibilidades de intervenção. Rev. Psicol. Saude. 2014;6(1):64-9

12. Caiuby AVS, Lacerda SS, Quintana MI, Torii TS, Andreoli SB. Adaptação transcultural da versão brasileira da Escala do Impacto do Evento - Revisada (IES-R). Cad. Saude Publica. [Internet]. 2012;28(3) [acesso em 15 jan 2014]. Disponível: http://dx.doi. org/10.1590/S0102-311X2012000300019

13. Reed SB. The remap process: measuring the emotional impact of an avent. Dallas Counseling \& Psychotherapy. [Internet] 2007 [acesso em 23 fev 2013]. Disponível: http://www.psychotherapy-center. com/Measuring_the_Impact_of_an_Event.html

14. Ministério da Saúde (BR). Conselho Nacional de Saúde. Diretrizes e normas regulamentadoras de pesquisa envolvendo seres humanos. Resolução n. 466, de 12 de dezembro de 2012. Brasília; 2012.

15. Perosa GB, Canavez IC, Silveira FCP, Padovani FHP, Peraçoli JC. Sintomas depressivos e ansiosos em mães 
de recém-nascidos com e sem malformações. Rev. Bras. Ginecol. Obstet. 2009;31(9):433-9.

16. Gomes LA, Torquato VS, Feitoza AR, Souza AR, Silva MAM, Pontes RJS. Identificação dos fatores de risco para depressão pós-parto: importância do diagnóstico precoce. Rev. Rene. 2010;11(n.esp):117-23.

17. Vieira AP, Almeida ACM, Souza JFD, Piccoli APB. Prevalência de depressão pós-parto em mulheres de um hospital universitário. Anuário de produção de iniciação cientifica discente. 2011;14(25):311-22.

18. Tahirkheli NN, Cherry AS, Tackett AP, McCaffree MA, Gillaspy SR. Postpartum depression on the neonatal intensive care unit: current perspectives. Int J Womens Health. [Internet] 2014; 6 [acesso em 01 dez 2014]. Disponível: http://www.ncbi.nlm.nih.gov/pmc/articles/ PMC4247145/

19. Daandels N, Arboit EL, Sand ICPVD. Produção de enfermagem sobre depressão pós-parto. Cogitare enferm. 2013;18(4):782-8.

20. Roecker S, Mai LD, Baggio SC, Mazzola JC, Marcon SS. The experience of mothers babies with malformation. Esc. Anna Nery. 2012;16(1):17-9.

21. Cantilino A, Zambaldi CF, Sougey EB, Rennó Jr J. Transtornos psiquiátricos no pós-parto. Arch. Clin. Psychiatry. 2010;37(6):278-84.

22. Santos RS, Dias IMV. Refletindo sobre a malformação congênita. Rev. Bras. Enferm. 2005;58(5):592-8.

23. Daugirdaite V, Van den Akker O, Purewal S. Posttraumatic Stress and Posttraumatic Stress Disorder after Termination of Pregnancy and Reproductive Loss: A Systematic Review. J. Pregnancy [Internet] 2015 [acesso em 23 fev 2015]. Disponível: http://dx.doi. org/10.1155/2015/646345 